\title{
Nebivolol Protects against Myocardial Infarction Injury via Stimulation of Beta 3-Adrenergic Receptors and Nitric Oxide Signaling
}

\author{
Zheng Zhang, Liping Ding, Zhitao Jin, Guojie Gao, Huijun Li, Lijuan Zhang, Lina Zhang, Xin Lu, Lihua Hu, \\ Bingwei Lu, Xiongjun Yu, Taohong Hu*
}

Department of Cardiology, The Second Artillery General Hospital of Chinese People's Liberation Army, Beijing, China

\begin{abstract}
Nebivolol, third-generation $\beta$-blocker, may activate $\beta 3$-adrenergic receptor (AR), which has been emerged as a novel and potential therapeutic targets for cardiovascular diseases. However, it is not known whether nebivolol administration plays a cardioprotective effect against myocardial infarction (MI) injury. Therefore, the present study was designed to clarify the effects of nebivolol on MI injury and to elucidate the underlying mechanism. MI model was constructed by left anterior descending (LAD) artery ligation. Nebivolol, $\beta 3-A R$ antagonist (SR59230A), Nitro-L-arginine methylester (L-NAME) or vehicle was administered for 4 weeks after MI operation. Cardiac function was monitored by echocardiography. Moreover, the fibrosis and the apoptosis of myocardium were assessed by Masson's trichrome stain and TUNEL assay respectively 4 weeks after MI. Nebivolol administration reduced scar area by $68 \%$ compared with MI group $(p<0.05)$. Meanwhile, nebivolol also decreased the myocardial apoptosis and improved the heart function after $\mathrm{Ml}$ ( $p<0.05 \mathrm{vs}$. MI). These effects were associated with increased $\beta 3-A R$ expression. Moreover, nebivolol treatment significantly increased the phosphorylation of endothelial NOS (eNOS) and the expression of neuronal NOS (nNOS). Conversely, the cardiac protective effects of nebivolol were abolished by SR and L-NAME. These results indicate that nebivolol protects against MI injury. Furthermore, the cardioprotective effects of nebivolol may be mediated by $\beta 3$-AR-eNOS/nNOS pathway.
\end{abstract}

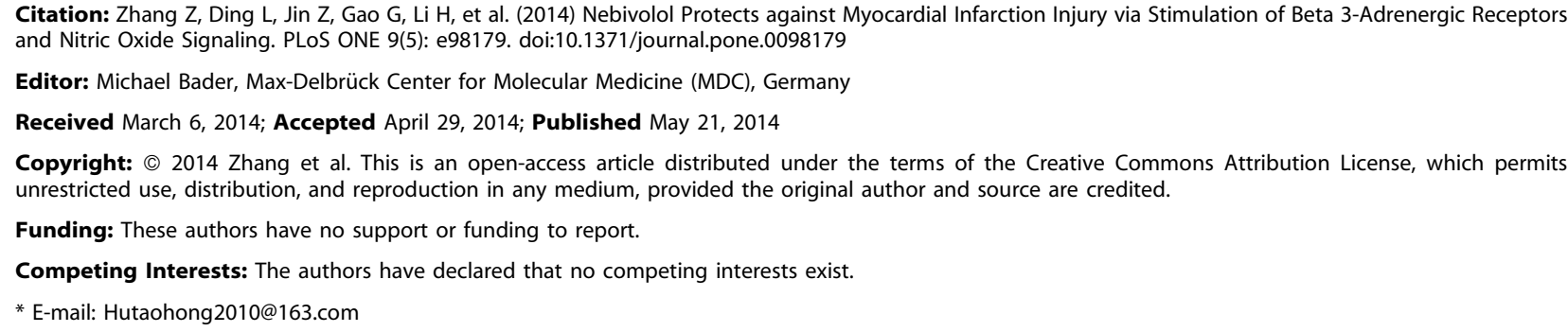

Citation: Zhang Z, Ding L, Jin Z, Gao G, Li H, et al. (2014) Nebivolol Protects against Myocardial Infarction Injury via Stimulation of Beta 3-Adrenergic Receptors and Nitric Oxide Signaling. PLoS ONE 9(5): e98179. doi:10.1371/journal.pone.0098179

Editor: Michael Bader, Max-Delbrück Center for Molecular Medicine (MDC), Germany

Received March 6, 2014; Accepted April 29, 2014; Published May 21, 2014

Copyright: (c) 2014 Zhang et al. This is an open-access article distributed under the terms of the Creative Commons Attribution License, which permits unrestricted use, distribution, and reproduction in any medium, provided the original author and source are credited.

Funding: These authors have no support or funding to report.

Competing Interests: The authors have declared that no competing interests exist.

*E-mail: Hutaohong2010@163.com

\section{Introduction}

Acute myocardial infarction (AMI), inducing permanent loss of cardiomyocyte mass and pathological left ventriclar remodeling, is one of the leading causes of death worldwide [1,2]. Accordingly, finding novel and effective therapies is important to reduce myocardial injury induced by MI. In the heart, $\beta$-adrenoceptors $(\beta-\mathrm{AR})$ are the primary regulator for cardiac performance, which are among the most widely used drugs for prevention and treatment of cardiovascular disease (eg, propranolol, which was the first $\beta$-blocker introduced into clinical practice) [3]. The cardioprotective effects of $\beta 1-$ and $\beta 2-\mathrm{AR}$ are well established, including negative chronotropic and inotropic effects. In contrast to the well-characterized $\beta 1 / \beta 2-\mathrm{AR}$, accumulating evidence revealed that $\beta 3-\mathrm{AR}$ also presents in the endothelium and myocardium [4]. Meanwhile, $\beta 3$-AR stimulation regulates specific effects in the cardiovascular system [5,6,7]. Experimental studies have shown the stimulation of the $\beta 3-\mathrm{AR}$ further activates endothelial nitric oxide synthase (eNOS) and increases NO release, which cause vasodilatation and improved endothelial function $[8,9]$. Furthermore, it is well established that $\beta 3-\mathrm{AR}$ is upregulated in failing hearts, which was associated with attenuated left ventricular (LV) hypertrophy [10]. Therefore, $\beta 3$-AR has been emerged as a potential target for the treatment of cardiovascular disease.
Nebivolol is the third-generation $\beta$-blocker approved by the Food and Drug Administration (FDA) for the treatment of hypertension. Given its greatest selectivity for cardiac $\beta 1$ adrenergic receptors without intrinsic sympathomimetic activity, nebivolol reduces systemic vascular resistance and improving diastolic function [11]. Therefore, nebivolol was shown to reduce mortality and morbidity in elderly patients with heart failure [12]. In addition to its $\beta 1$-blocking properties, previous studies have demonstrated that nebivolol also exhibits vasodilating properties by stimulating $\beta 3$-AR [13]. Studies also confirm that $\beta 3$-AR further activates nitric oxide synthase (eNOS) and increases NO release which exerts profound cardioprotective effects [14].

Although previous studied indicated that nebivolol could reduce cardiac remodeling and preserve cardiac function through $\beta 3-\mathrm{AR}$ pathway, it is not known whether nebivolol administration plays a cardioprotective effect against MI injury. Therefore, we designed the present study to explore the potential role of nebivolol during MI and to elucidate the underline mechanism of their protective effects.

\section{Methods}

Animals

One hundred and fifty adult C57BL6/J mice (male, weighing 22 to $25 \mathrm{~g}$ ) were housed in a temperature-controlled animal facility 
with a 12-h light/dark cycle. All procedures were approved by the Second Artillery General Hospital of Chinese People's Liberation Army Committee on Animal Care. (Approval ID: 2012-04) and were in compliance with Guidelines for the Care and Use of Laboratory Animals, as published by the National Academy Press. Mice were euthanized by cervical dislocation after anesthesia with $5 \%$ isoflurane.

Mice were randomly allocated into 5 groups with $\mathrm{n}=30$ each: (1) sham group (Sham); (2) MI group (MI); (3) MI + Nebivolol group (Nebivolol); (4) MI + Nebivolol+SR59230A group (Nebivolol+SR); (5) MI + Nebivolol+ L-NAME group (Nebivolol+LNAME). After MI model, all of the above drugs or vehicles were administrated for 4 weeks respectively. Blood pressure was monitored with a tailcuff system (see Methods S1).

\section{Myocardial infarction model construction and Nebivolol administration}

Myocardial infarction (MI) model was constructed by left anterior descending (LAD) artery ligation as previous described [15]. In brief, mice underwent aseptic lateral thoracotomy after anesthetized with $2 \%$ isoflurane. LAD was permanently ligated with a 6-0 suture. The ligation was deemed successful by characteristic ECG changes. Sham operated control mice underwent the same surgical procedures except that the suture placed under the left coronary artery was not tied.

Mice of MI+ Nebivolol group were administrated with Nebivolol at $2 \mu \mathrm{g} / \mathrm{kg} /$ hour via osmotic mini-pumps (Alzet Inc, Cupertino, CA) one day after MI operation. Mice treated with $\beta 3$ $\mathrm{AR}$ antagonist were administrated with SR59230A at $0.1 \mathrm{mg} / \mathrm{kg}$ / hour via another osmotic mini-pump. Moreover, mice from Nebivolol+L-NAME group were treated with Nitro-L-arginine methylester (L-NAME) by intraperitoneal injection at $25 \mathrm{mg} / \mathrm{kg}$ for 4weeks after LAD ligation.

\section{Postmortem Histological determination of scar area}

Mice were euthanized by cervical dislocation after anesthesia with 5\% isoflurane for histological assay at 4 weeks after MI [15]. Hearts were embedded in paraffin after being fixed in $4 \%$ paraformaldehyde. Serial sections (5 $\mu \mathrm{m}$ thickness) were performed Masson's trichrome stain to detect scar area and fibrosis in cardiac muscle. Computerized morphometry was used to calculate the scar extent as the ratio of scar and total left ventricular area using Imaging Pro Plus software.

\section{Echocardiographic studies of cardiac function}

Echocardiography was performed to assess the cardiac function after MI in a blinded manner [16]. At 2 days post operation (POD) and weekly until sacrificed, Mice were anesthetized ( $2 \%$ isoflurane and oxygen) and put in a supine position. Both two-dimensional and $\mathrm{M}$-mode images were recorded using a $30-\mathrm{MHz}$ transducer. Left ventricular systolic dimension (LVDs), left ventricular diastolic dimension (LVDd), anterior wall thickness (AWT) and posterior wall thickness (PWT) were measured to calculate left ventricular ejection fraction (LVEF) and fractional shortening (FS) as an average of three beats.

\section{In vitro apoptosis assay}

Terminal deoxynucleotidyl transferase-mediated dUTP-biotin nick end labeling (TUNEL) assay was performed to evaluate myocardial apoptosis [15]. In brief, serial sections of heart tissue were stained with fluorescein-dUTP (In Situ Cell Death Detection Kit; Roche Diagnostics) for apoptotic cell according to the manufacturer's instructions. To identify all cell nuclei and myocardium, additional staining was performed using $4^{\prime}, 6$ diamidino-2-phenylindole (DAPI) (Sigma) and the monoclonal antibody against Troponin I (cTnI, Santa Cruz). Randomly selected microscopic fields $(n=5)$ were evaluated to calculate the apoptotic index which was termed as the ratio of apoptosis (TUNEL positive) cells to total cells

\section{Western blot assay}

4 weeks after cell transplantation, hearts were excised from periinfarcted areas in LV for Western blotting assay following standard protocol [17]. Protein was isolated from homogenized LV tissue using cell lysis buffer (Cell Signaling Technology, Danvers, MA). Equal amounts of heated protein $(100 \mu \mathrm{g})$ were separated by electrophoresis on 12\% SDS-PAGE gels in a Tris/HCl buffer system, and sequentially electrophoretically transferred to nitrocellulose (NC) membranes. After blocking with 5\% nonfat dry milk in Tris-buffered saline containing $0.05 \%$ Tween-20 (TBST), NC membranes were subjected to immunoblotting with appropriate primary antibodies at $4{ }^{\circ} \mathrm{C}$ over night. Immunoreactivity was visualized by incubation with appropriate horseradish peroxidaseconjugated secondary antibodies and enhanced chemiluminescence kit (Amersham Bioscience, Buchinghamshire, UK). The densitometric analysis of Western blots was carried out using VisionWorks LS, version 6.7.1.

The following primary antibodies were used: $\beta 1$-adrenergic receptor (1:1000, Abcam); $\beta 2$-adrenergic receptor (1:1000, Abcam); $\beta 3$-adrenergic receptor (1:1000, Abcam); eNOS (1:500,Cell Signaling Technology); Phospho-eNOS at Ser1177,Thr495, Ser114 (p-eNOS Ser1177, p-eNOS Thr495, peNOS Ser1141:500, Cell Signaling Technology); iNOS (1:1000, Cell Signaling Technology); nNOS (1:1000, Cell Signaling Technology) and $\beta$-actin (1: 5000, Abcam).

\section{Statistical analysis}

All data are presented as mean \pm SEM. Statistics were calculated using Prism 5.0 (GraphPad Software Inc, San Diego, CA, USA). Statistical comparisons for different groups were performed using one-way ANOVA followed by Student's paired, two-tailed t test for two groups' comparison. $\mathrm{P}$ values $<0.05$ were considered statistically significant.

\section{Results}

\section{Nebivolol limits the extent of myocardial injury and decreases the mortality following $\mathrm{MI}$}

To study the effects of nebivolol on the extent of fibrosis after MI, we performed Masson's trichrome staining. As illustrated in Figures 1a, severe fibrosis was observed in the hearts of MI group which was decreased by nebivolol administration. Quantitative measurement revealed that the scar area in MI group was $50.32 \pm 4.67 \%$. Conversely, the scar area in mice receiving nebivolol was $15.75 \pm 4.75 \%$, significantly less than that in MI group $(15.75 \pm 1.75 \%$ vs. $50.32 \pm 2.67 \%, \mathrm{P}<0.05)$.

Furthermore, echocardiogram studies were performed to evaluate the cardiac function after MI in all groups. Serial echocardiographic analysis indicating that nebivolol administration manifested a trend towards improvement of cardiac performance after MI. Compared with sham group, MI increased the LVEDd and LVESd which were abolished by nebivolol treatment (Fig. 1c and 1d). Furthermore, the LVEF and FS were significantly enhanced in nebivolol group compared with MI group (Fig. le and 1f). These data suggested that nebivolol administration decreased fibrosis and preserved cardiac function after MI. Meanwhile, MI decreased the blood pressure, whereas 
A

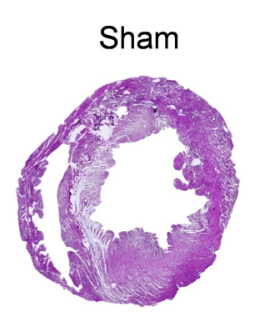

MI

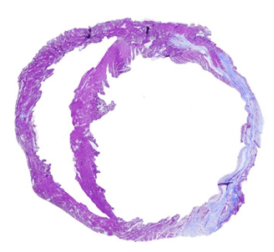

B

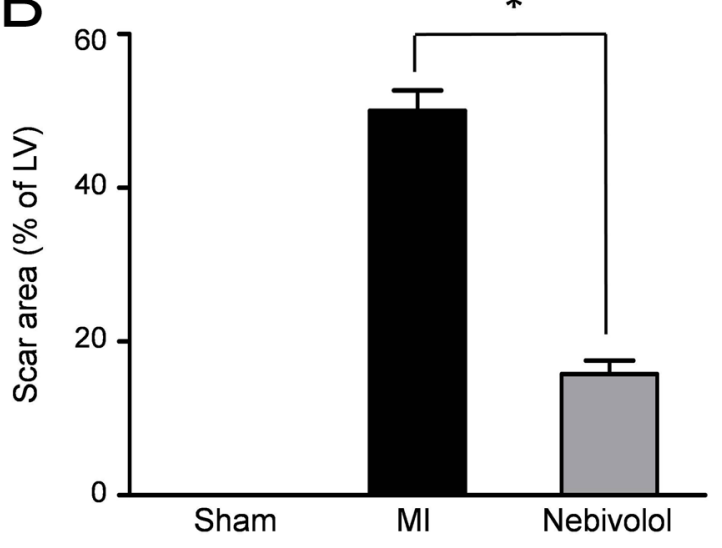

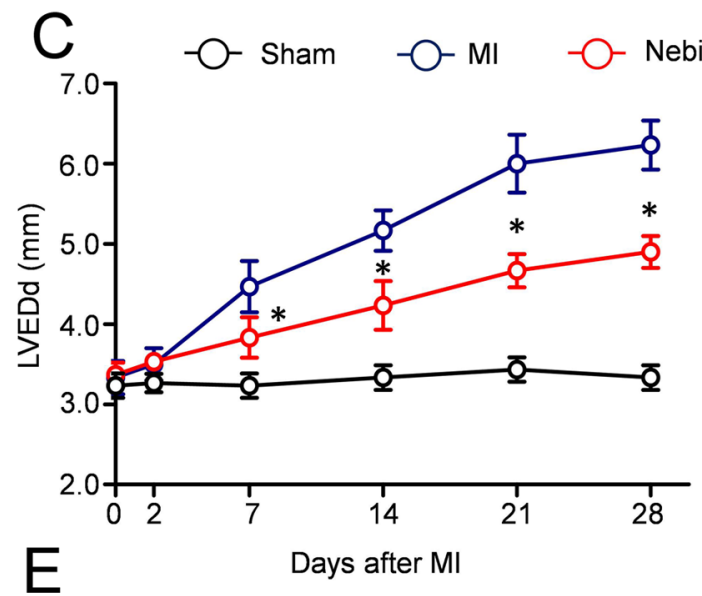

D
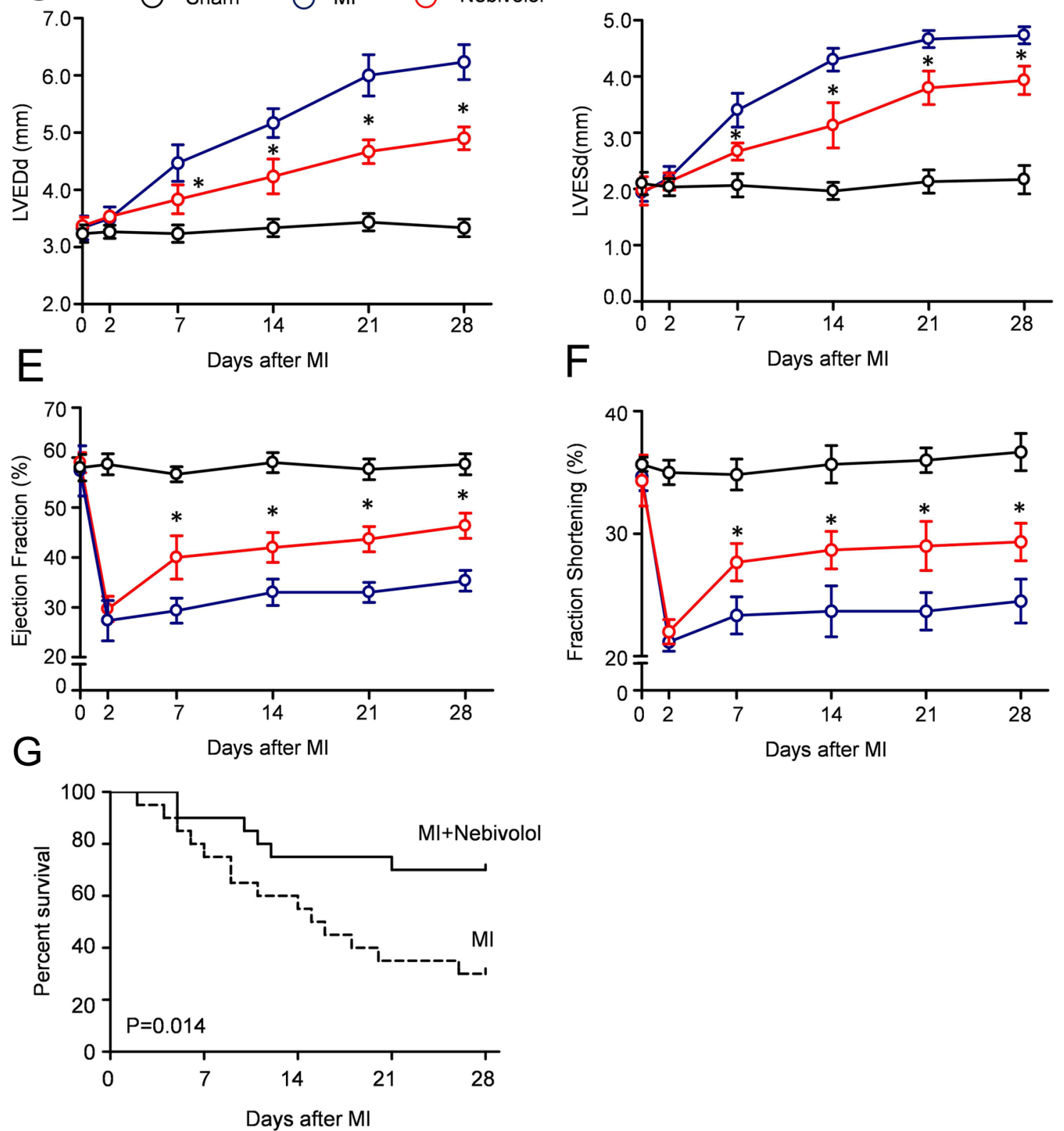
Figure 1. Effects of nebivolol on left ventricular fibrosis and heart function after MI. (a) Representative Masson's trichrome staining revealed left ventricular fibrosis 4 weeks after MI (magnification: 4x). (b) Quantitative analysis of the scar area $\left(n=10,{ }^{*} p<0.05\right)$. Quantification of left ventricular end diastolic diameter (LVEDd) (c), end systolic diameter (LVESd) (d), left ventricular ejection fraction (EF) (e) and fractional shortening (FS) (f) $\left(\mathrm{n}=30,{ }^{*} p<0.05\right.$ vs. MI). Kaplan-Meier survival curves in Ml group and Ml+ nebivolol group (g).

doi:10.1371/journal.pone.0098179.g001

increased the heart rate. However, nebivolol administration significantly increased the blood pressure, with decreased heart rate, compared MI group (Table S1). Moreover, the KaplanMeier survival curves (Fig.2g) indicated that nebivolol administration significantly decreased the mortality after MI.

\section{Nebivolol reduced cardiomyocyte apoptosis after MI}

We next performed TUNEL staining to confirm the protective effect of nebivolol on the apoptosis of cardiomyocytes induced by MI. Representative immunofluorescence results in figure 2a revealed that apoptotic cardiomyocytes were more frequently observed in the MI group compared with Sham group or Nebivolol group. Quantitative analyses (Fig. 2b) demonstrated that the apoptosis index in MI group was $43.67 \pm 4.33 \%$, significantly more than that in Sham group $(6.54 \pm 0.89 \%, \mathrm{P}<$ $0.05)$. Conversely, nebivolol administration decreased the apoptosis of cardiomyocyte induced by $\mathrm{MI}(28.33 \pm 1.76 \%, \mathrm{P}<0.05$ vs. MI).

\section{Nebivolol enhanced cardiac $\beta 3-A R$ expression after $\mathrm{Ml}$}

Western blotting assay was performed to investigate the cardiac expressions of $\beta$-adrenoreceptor, including $\beta 1 / \beta 2-\mathrm{AR}$ and $\beta 3-\mathrm{AR}$. Representative bloting results (Fig. 3a) indicated that the expression of $\beta 3-\mathrm{AR}$ was decreased after MI which was abolished by nebivolol treatment. Semiquantitative analyses revealed that no changes in the expressions of $\beta 1-\mathrm{AR}$ and $\beta 2-\mathrm{AR}$ were observed in all groups (Fig. 3b,c). Furthermore, the expression ratio of $\beta 3-\mathrm{AR}$ to $\beta$-actin in MI group was $14.80 \pm 2.27 \%$, significantly decreased compared with Sham group $(28.03 \pm 2.06 \%, \mathrm{P}<0.05)$. Conversely, nebivolol treatment increased $\beta 3-\mathrm{AR}$ expression $(33.40 \pm 1$. $78 \%, \mathrm{P}<0.05$ vs. MI) (Fig. 3d). These data indicated that the protective effect of nebivolol may be mediated by $\beta 3-\mathrm{AR}$.

\section{Nebivolol enhanced eNOS activation and increased nNOS protein expression}

Previous studies suggested that $\beta 3-\mathrm{AR}$ stimulation results in NO production via NOS which includes three isoforms (i.e. eNOS, nNOS, and iNOS). Therefore, we further performed Western blot assay to evaluate the effect of nebivolol on the expressions of NOS isoforms after MI. Representative bloting results and semiquantitative analyses (Fig. 4a-d) demonstrated that total eNOS and iNOS were unchanged in all groups. Conversely, the expression of nNOS was significantly decreased in MI group which was abolished by nebivolol treatment.

The activity of eNOS is generally modulated by three phosphorylation sites. Phosphorylation at Ser1177 activates eNOS, whereas phosphorylations at Serl14 and Thr 497 inhibit the eNOS activity.To furtherly investigate the role of nebivolol on the activation of eNOS, we examined the expressions of phospheNOS (p-eNOS). As representative results (Fig. 4a) and semiquantitative analyses in figure $4 \mathrm{e}-\mathrm{f}$, the expression of $\mathrm{p}$ $\mathrm{eNOS}^{\mathrm{Ser} 1177}$ significantly decreased, whereas the expression of $\mathrm{p}$ eNOS $^{\text {Thr495 }}$ increased in MI group compared with Sham group. Furthermore, nebivolol administration resulted in an increase in peNOS $^{\text {Ser1177 }}$ expression with decreased eNOS phosphorylations at Thr 497 compared with MI. Interestingly, no difference of peNOS $^{\text {Ser114 }}$ expression was observed in all group (Fig. 4g).
A
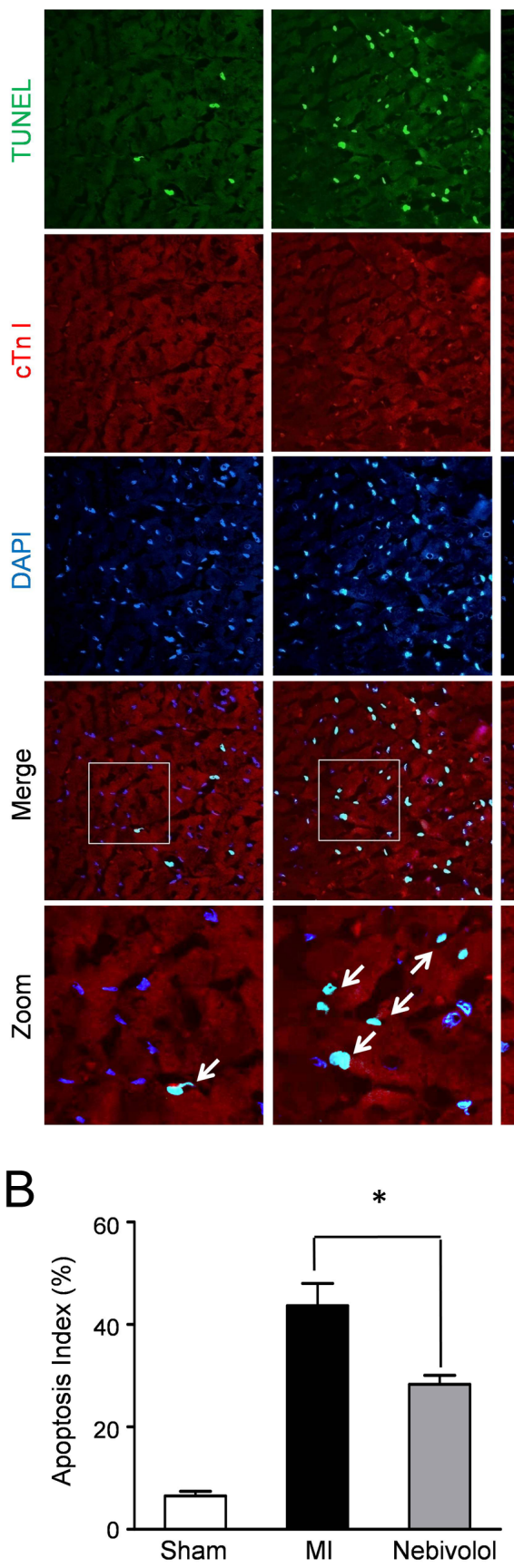

Figure 2. Nebivolol administration decreased cardiomyocyte apoptosis. (a) Representative photographs of TUNEL-stained heart sections from sham group, $\mathrm{Ml}$ group and $\mathrm{Ml}+$ nebivolol group. Apoptotic nuclei were identified as TUNEL positive (green fluorescent). Myocardium was stained using a monoclonal antibody against Troponin I (red fluorescent) and total nuclei was stained by DAPI (blue fluorescent). Scale bar represents $50 \mu \mathrm{m}$. (b) Apoptotic cells were quantified by apoptotic index (Al) which was termed as the percentage of apoptotic cells. $\left(n=10,{ }^{*} p<0.05\right)$.

doi:10.1371/journal.pone.0098179.g002 

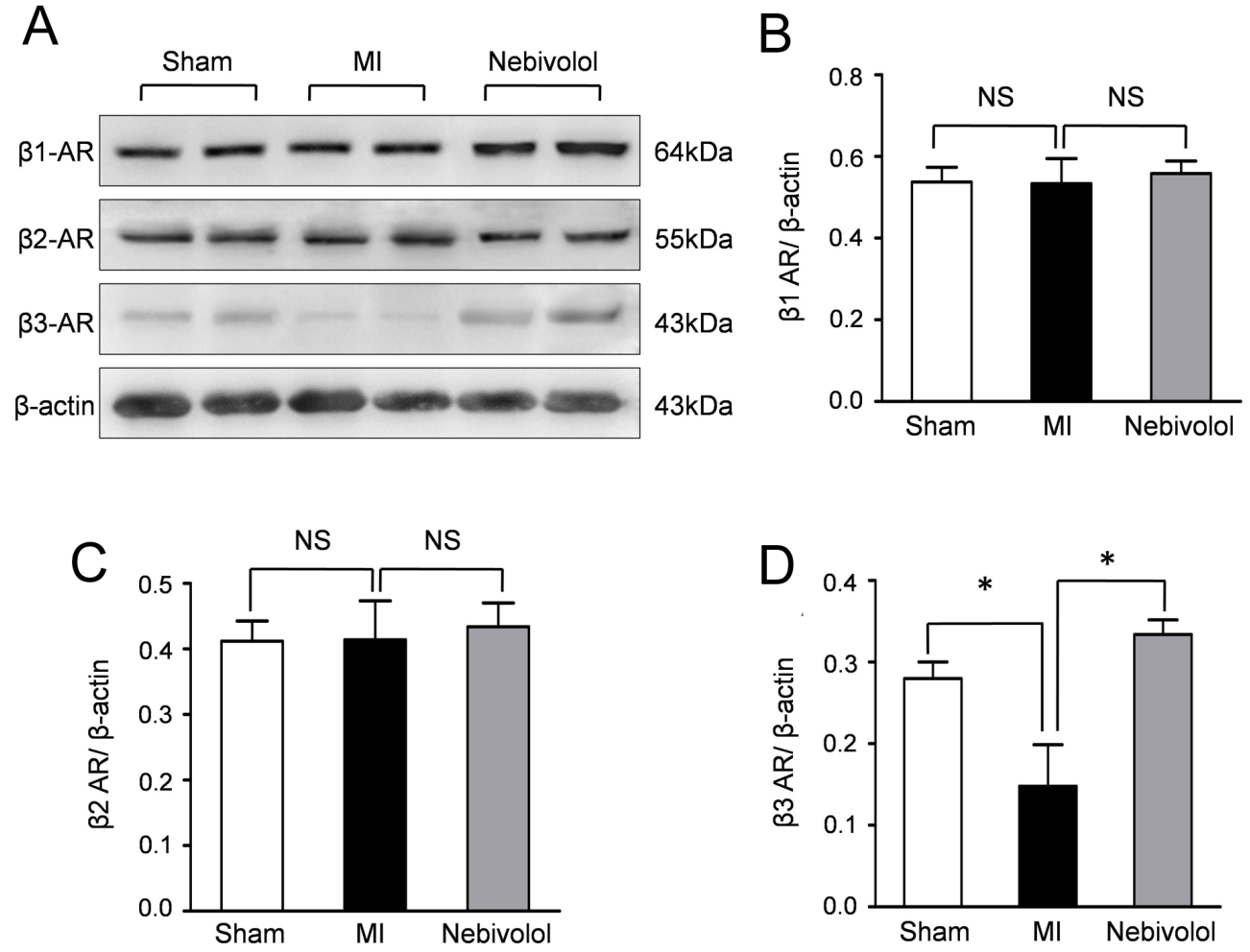

Figure 3. Effect of nebivolol on the expressions of $\boldsymbol{\beta}$-AR subtypes. (a) Representative immunoblots of $\beta 1-A R$, $\beta 2-A R$ and $\beta 3-A R$ in sham group, $M I$ group and $M I+$ nebivolol group. The semiquantitative analysis of the expressions of $\beta 1-A R(b), \beta 2-A R(c)$ and $\beta 3-A R(d)(n=10, N S$ : non significance, $\left.{ }^{*} p<0.05\right)$.

doi:10.1371/journal.pone.0098179.g003

\section{Cardiacprotective effects of nebivolol was abolished by $\beta 3-A R$ antagonism and NOS inhibition}

SR59230A and L-NAME are well known as the inhibitors of $\beta 3-\mathrm{AR}$ and NOS respectively. To illuminate the mechanism of the protective effect of nebivolol, either SR59230A or L-NAME was administrated with nebivolol. Representative Masson's trichrome staining and quantitative measurement in figures 5a,b revealed that the scar area in Nebivolol+SR group and Nebivolol+LNAME group were $49.25 \pm 1.75 \%$ and $49.02 \pm 2.05 \%$ respectively, significantly higher than that in Nebivolol group $(19.75 \pm 1.25 \%$, $\mathrm{P}<0.05)$. Subsequently, echocardiogram studies were performed to evaluate the cardiac function (Fig. 5c-f). Serial echocardiographic analysis indicating that the LVEDd and LVESd in Nebivolol+SR group and Nebivolol+L-NAME group were increased, whereas the LVEF and FS were significantly decreased compared with that in Nebivolol group. These data suggested that the cardioprotective effects of nebivolol were abolished by specific inhibition of $\beta 3-\mathrm{AR}$ and NOS.

NOS inhibition and $\beta 3-A R$ antagonism abolished the antiapoptosis effect of nebivolol

Representative TUNEL staining in figure 6 a revealed that apoptotic cardiomyocytes were more frequently observed in Nebivolol+SR group and Nebivolol+L-NAME group compared with Nebivolol group. Quantitative analyses (Fig. 6b) demonstrated that the apoptosis index in Nebivolol+SR group and Nebivolol+L-NAME group were $44.67 \pm 1.76 \%$ and $48.33 \pm 1.85 \%$ respectively, significantly higher than that in Nebivolol group $(28.33 \pm 1.65 \%, \mathrm{P}<0.05)$.
Nebivolol induced $\beta 3$-AR signaling activation was abolished by SR59230A and L-NAME

Representative bloting results and semiquantitative analyses (Fig. 7a-c) indicated that the expressions of $\beta 3-\mathrm{AR}$ were decreased in MI+Nebivolol+SR group compared with that in MI+Nebivolol group. However, NOS inhibition with L-NAME had no effect on the expression of $\beta 3-\mathrm{AR}$. Furthermore, the expressions of nNOS in MI+Nebivolol+SR group and MI+Nebivolol+L-NAME group were significantly decreased compared with that in MI+Nebivolol group. The phosphorylations of eNOS were also detected by Western bolt assay. As representative results and semiquantitative analyses (Fig. 7d-f), the expressions of $\mathrm{p}$-eNOS ${ }^{\text {Ser1177 }}$ were significantly decreased in MI+Nebivolol+SR group and MI+ Nebivolol+L-NAME group. Conversely, SR59230A or L-NAME administrated resulted in a significantly increase of $\mathrm{p}^{-\mathrm{NONS}}{ }^{\mathrm{Thr} 495}$ expression. Moreover, no difference of $\mathrm{p}-\mathrm{eNOS}^{\mathrm{Ser} 114}$ expressions was observed in three groups.

\section{Discussion}

In the present study, we found that nebivolol decreased the apoptosis of cardiomyocytes, and enhanced cardiac function after MI injury. Furthermore, the protective effects of nebivolol were abolished by $\beta 3-\mathrm{AR}$ inhibition. Overall, this study demonstrates that nebivolol prevents cardiac dysfunction induced by MI via $\beta 3$ AR-eNOS/nNOS pathway.

Nebivolol has recently been shown to cause peripheral vasodilatation, and provide beneficial effects on cardiac dysfunction. Sorrentino et al. [18] observed a significant increased EF\% after treatment with nebivolol for 30 days after myocardial I/R injury. By contrary, Lefer et al. [19] failed to demonstrate 


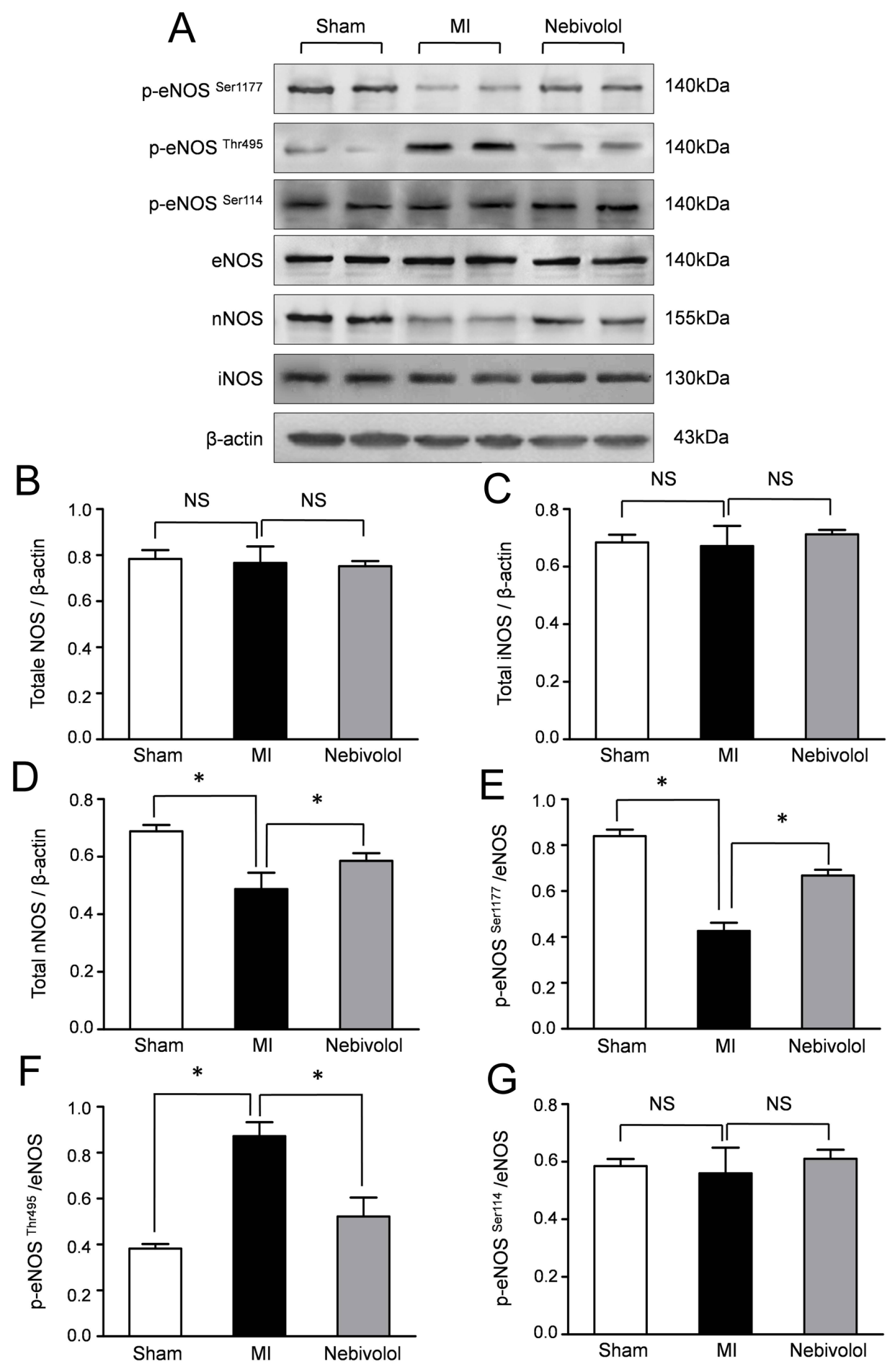

Figure 4. Nebivolol administration altered the phosphorylation status of eNOS and increased the expression of $\mathrm{nNOS}$. (a) Representative immunoblots of $p$-eNOS (Ser1177/Thr495/Ser114), total eNOS, nNOS and iNOS in sham group, Ml group and Ml+ nebivolol group.

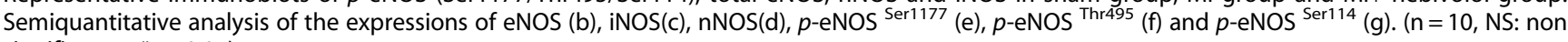
significance, $\left.{ }^{*} p<0.05\right)$.

doi:10.1371/journal.pone.0098179.g004

significant improvements in cardiac function post-reperfusion after nebivolol treatment. In the current study, we confirm that nebivolol administration decreased the extent of fibrosis and reduced the apoptosis of cardiomyocyte, leading to an enhanced cardiac function recovery. Moreover, nebivolol administration decreased the mobility after MI. These results indicated that nebivolol exerts a cardioprotective role in the failing heart induced by MI.

The $\beta$-adrenoceptors, belonging to the $G$ protein-coupled receptor super family, play an essential role in cardiovascular 
A

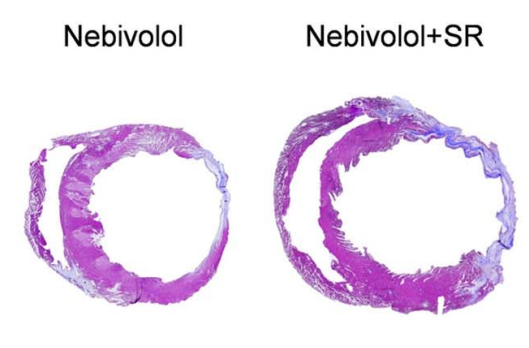

Nebivolol+L-NAME

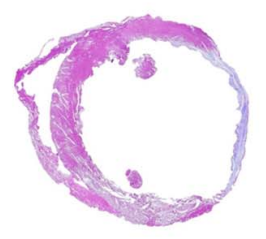

B

Nebivolol

Nebivolol+SR

Nebivolol+L-NAME

\footnotetext{
$\mathrm{D}$
}

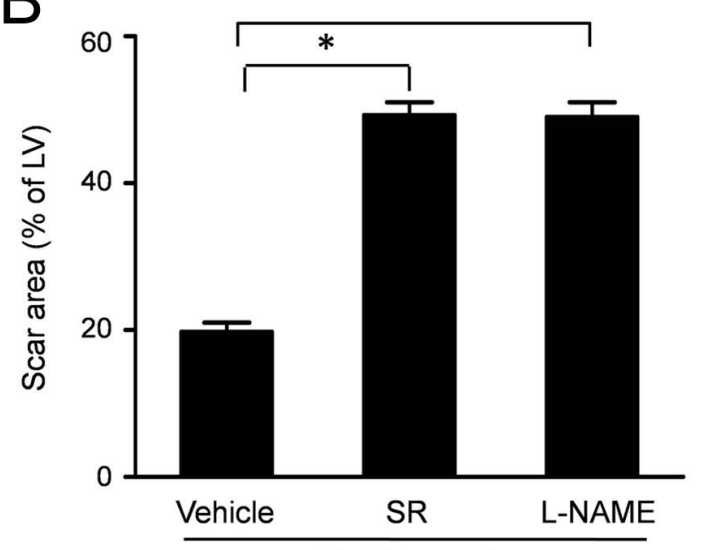

Ml+Nebivolol

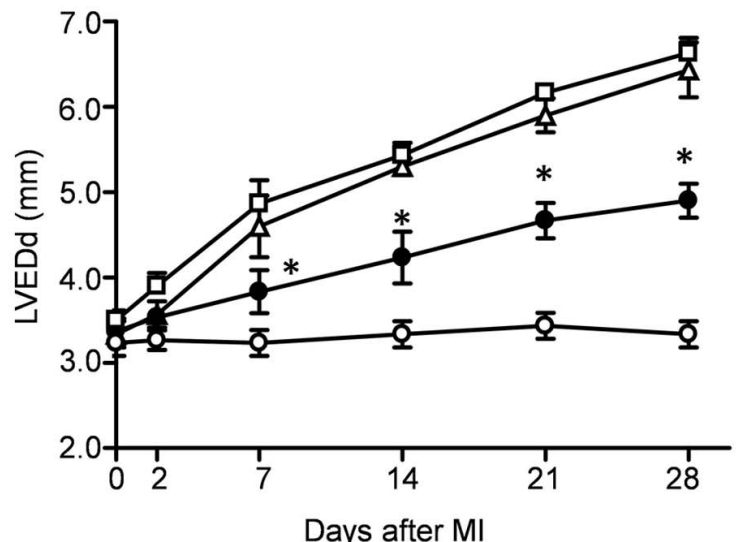

$E$

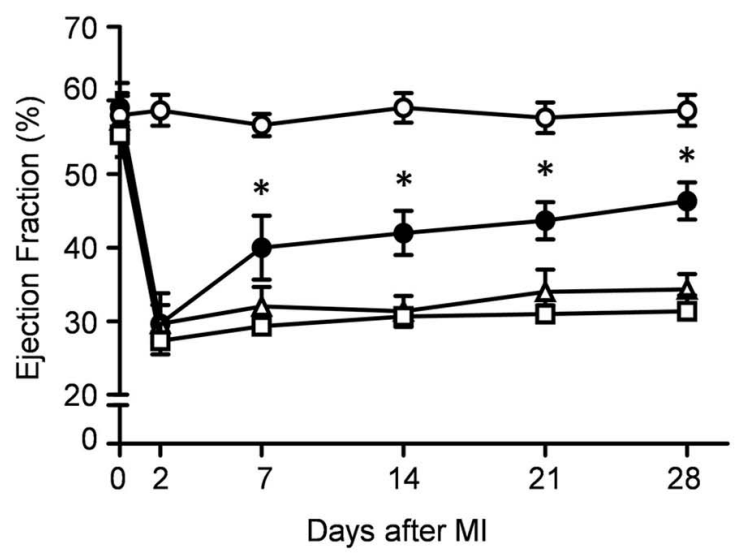

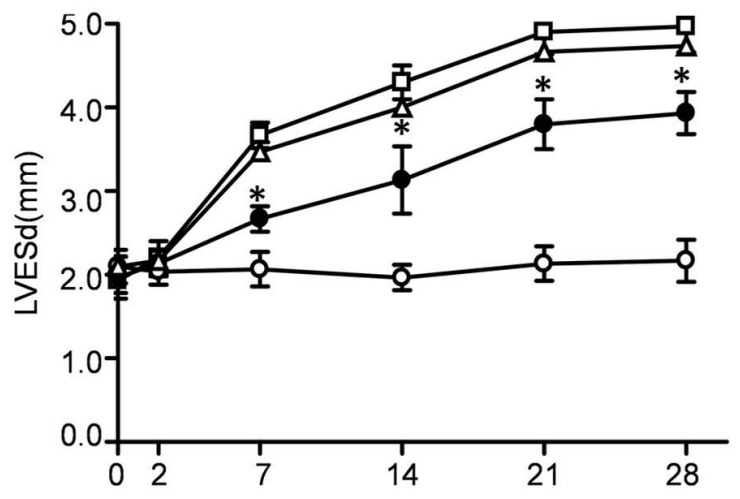

Days after MI

$\mathrm{F}$

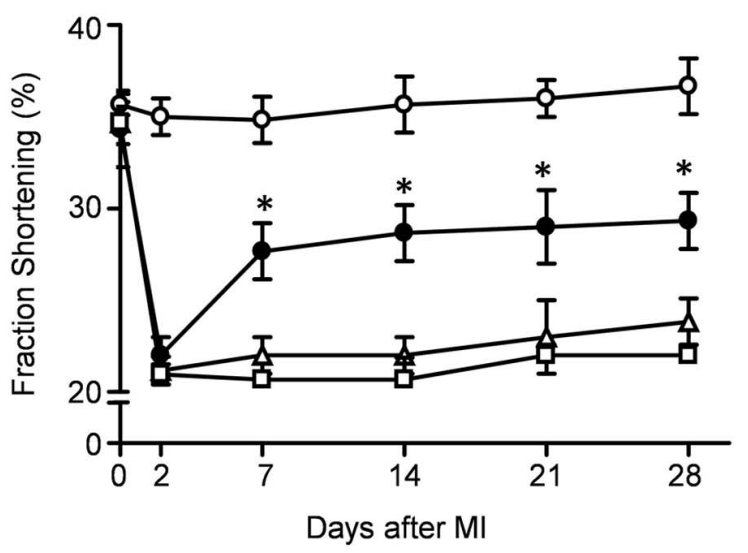

Figure 5. Cardiacprotective effects of nebivolol was abolished by SR59230A or L-NAME. (a) Representative Masson's trichrome staining 4 weeks after MI in Nebivolol group, Nebivolol+SR group and Nebivolol+L-NAME group (magnification: 4x). (b) Quantitative analysis of the scar area $\left(n=10,{ }^{*} p<0.05\right)$. Serial echocardiographic analysis was performed to assess the heart function in all groups, including left ventricular end diastolic diameter (LVEDd) (c), end systolic diameter (LVESd) (d), left ventricular ejection fraction (EF) (e) and fractional shortening (FS) (f). ( $\mathrm{n}=30,{ }^{*} p<0.05 \mathrm{vs}$. MI.)

doi:10.1371/journal.pone.0098179.g005

function. The $\beta 1$ - and $\beta 2-\mathrm{ARs}$, which mediates chronotropic and inotropic effects, are well established. Although the precise cardiac effects remain uncertain, $\beta 3-\mathrm{AR}$ is of increasing interest due to its potential as a potential target for cardiovascular disease treatment [8]. Accumulating evidence suggests that $\beta 3-\mathrm{AR}$ is activated at higher concentrations of catecholamines, and plays a negative inotropic effect antagonizing the effects of $\beta 1 / 2-A R s$ [20]. Moreover, previous study indicated that $\beta 3$-AR plays a protective effect on exacerbated hypertrophy and cardiac systolic dysfunction induced by pressure-overload [21,22]. All these literature support 


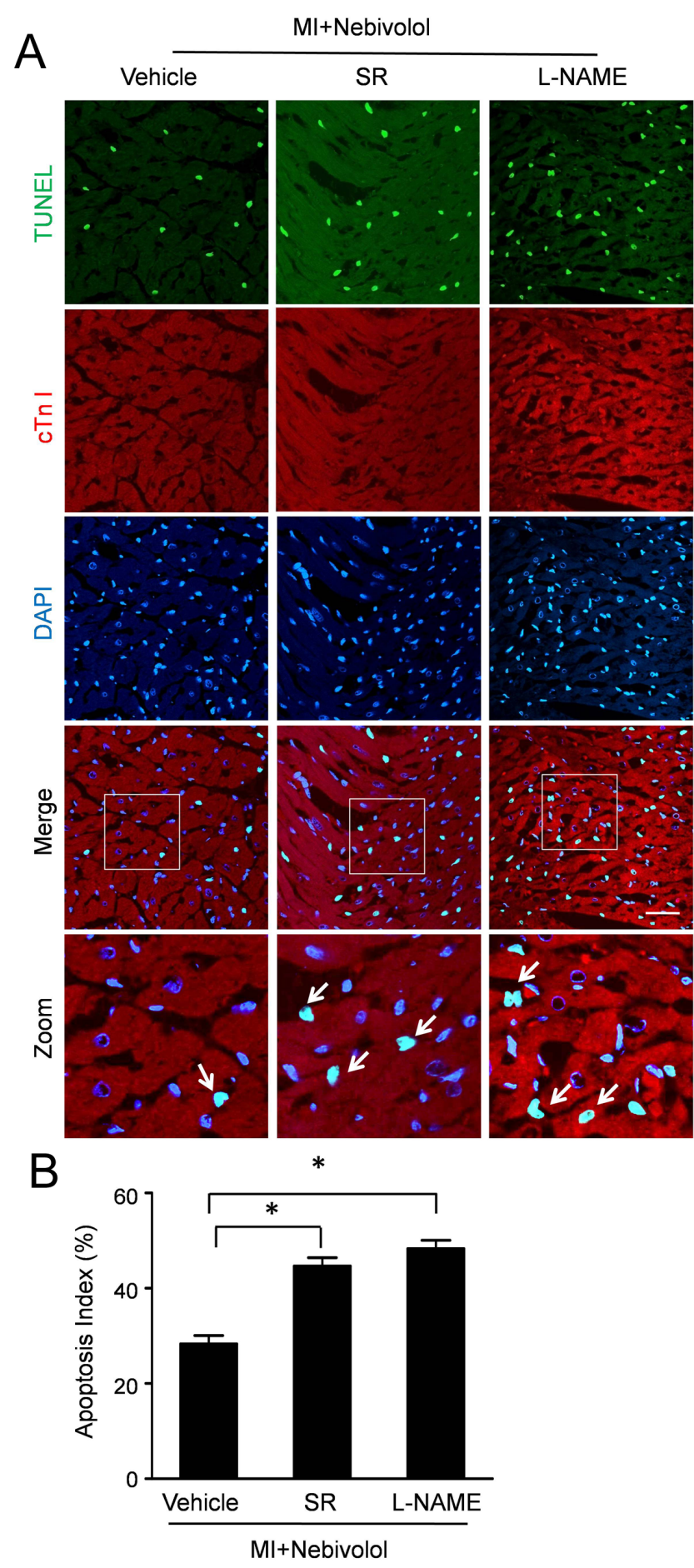

Figure 6. Anti-apoptosis effect of nebivolol was abolished by SR59230A or L-NAME. (a) Representative photographs of TUNELstained heart sections from Nebivolol group, Nebivolol+SR group and Nebivolol+L-NAME group (Scale bar: $50 \mu \mathrm{m}$ ). (b) Apoptotic cells were quantified by apoptotic index (Al) which was termed as the percentage of apoptotic cells. $\left(n=10,{ }^{*} p<0.05\right)$.

doi:10.1371/journal.pone.0098179.g006

a significant role for $\beta 3-\mathrm{AR}$ in the modulation of cardiac remodeling after MI.

Previous studies suggested that the protective effects of nebivolol are dependent on $\beta 3$-AR stimulating [19]. However, the direct in vivo evidence has been lacking. In the present study, we observed that expression of $\beta 3-\mathrm{AR}$ was decreased in the infarcted heart and nebivolol treatment increased $\beta 3$-AR expression. Furthermore, the cardiacprotective effects of nebivolol were abolished by $\beta 3$-AR antagonism SR59230A, indicated that $\beta 3$-AR was involved in the protective effects of nebivolol.

Although the mechanism is not clarified, previous studies have demonstrated that the protective effects of $\beta 3-\mathrm{AR}$ stimulation were inhibited by NOS inhibitor and could be reversed by excess NO production [9]. This evidence indicated that $\beta 3-\mathrm{AR}$ plays a protective role by activating NOS pathway and NO release. However, three NOS isoforms (i.e. eNOS, nNOS, and iNOS) are involved in $\mathrm{NO}$ release which is involved in the regulation of myocardial function. There remains great controversy over which NOS isoform is the chief player in $\beta 3$-AR signaling. Several studies have suggested that endothelial NOS (eNOS) is solely responsible for $\beta 3-\mathrm{AR}$ induced negative inotropy. However, previous reports also revealed that the nNOS expression and nNOS-derived NO levels are both increased in failing hearts [8]. Moreover, it is an apparent paradox between the observations that eNOS-derived NO is decreased in failing myocardium, indicating the relative involvement of neuronal NOS (nNOS) in $\beta 3-\mathrm{AR}$ modulates NO signaling.

Three phosphorylation sites, serine residue 1177 (Ser1177),threonine residue 497 (Thr495) and serine residue 114(Ser114), modulate eNOS activity. Phosphorylation at Ser1177 activates eNOS, whereas phosphorylations at Ser114 and Thr 497 inhibit the eNOS activity [23]. In the present study, we found that nebivolol treatment significantly increased p-eNOS ${ }^{\text {Ser1177 }}$ and decreased p-eNOS ${ }^{\text {Thr495 }}$ in the infarcted heart. Interestingly, the phosph-eNOS at Serl14 was unaltered with or without nebivolol administration, which was consistent with previous studies. The alterations of eNOS phosphorylation suggested that MI inhibited the eNOS activation which was abolished by nebivolol treatment. Emerging evidence demonstrated that nNOS-derived NO production plays a substantial role in regulating myocardial contraction [24]. Our results demonstrated that the expression of nNOS was decreased after MI which was abolished by nebivolol treatment. Consistently, previous studies revealed that administration of nebivolol or select $\beta 3$-AR agonist CL 316243 both increased the expression of $\mathrm{nNOS}$ and reduced myocardial $\mathrm{I} / \mathrm{R}$ injury.

Our study provides evidence that nebivolol treatment enhanced eNOS activation and increased nNOS protein expression, which was abolished by $\beta 3-\mathrm{AR}$ antagonism SR59230A and NOS inhibitors L-NAME respectively. Moreover, nebivolol administration had no effect on the expression of iNOS. These results indicated that nebivolol acts as a dual activator of eNOS and nNOS to generate NO in the myocardium. Furthermore, the cardiacprotective effects of nebivolol were also abolished by LNAME, indicated that nebivolol prevents cardiac dysfunction induced by MI by eNOS and nNOS which was activated by $\beta 3$ AR pathway.

Although our study bears some clinical relevance, there are many limitations. First, the detailed physiologic and pathologic functions of $\beta 3-\mathrm{AR}$ have not been extensively characterized. Moreover, there are still intense controversies about which NOS isoform is the chief player in $\beta 3$-AR signaling. Further studies defining the exact mechanism are needed. In conclusion, we provided evidence that the administration of nebivolol has a substantial effect on recovery of heart function by eNOS and nNOS activated by $\beta 3-\mathrm{AR}$. These data collectively indicate that nebivolol administration has practical clinical use following myocardial infarction. 
A
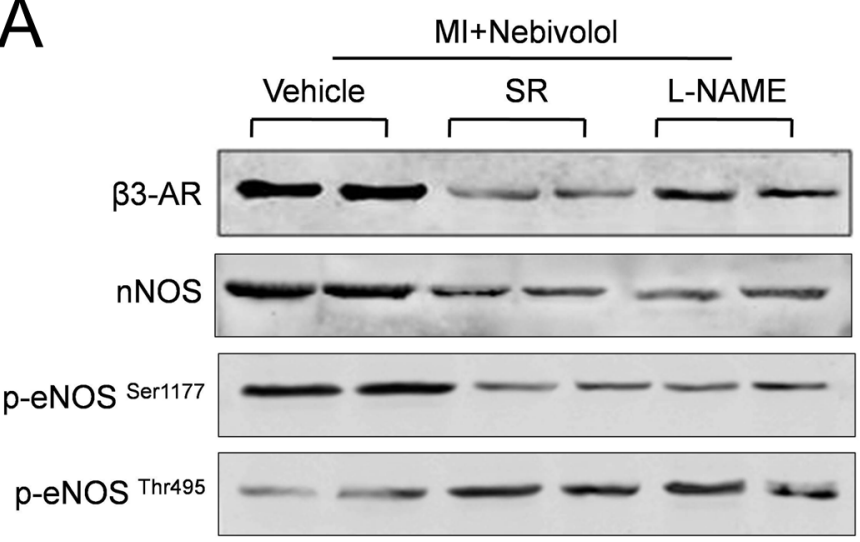

p-eNOS Ser114
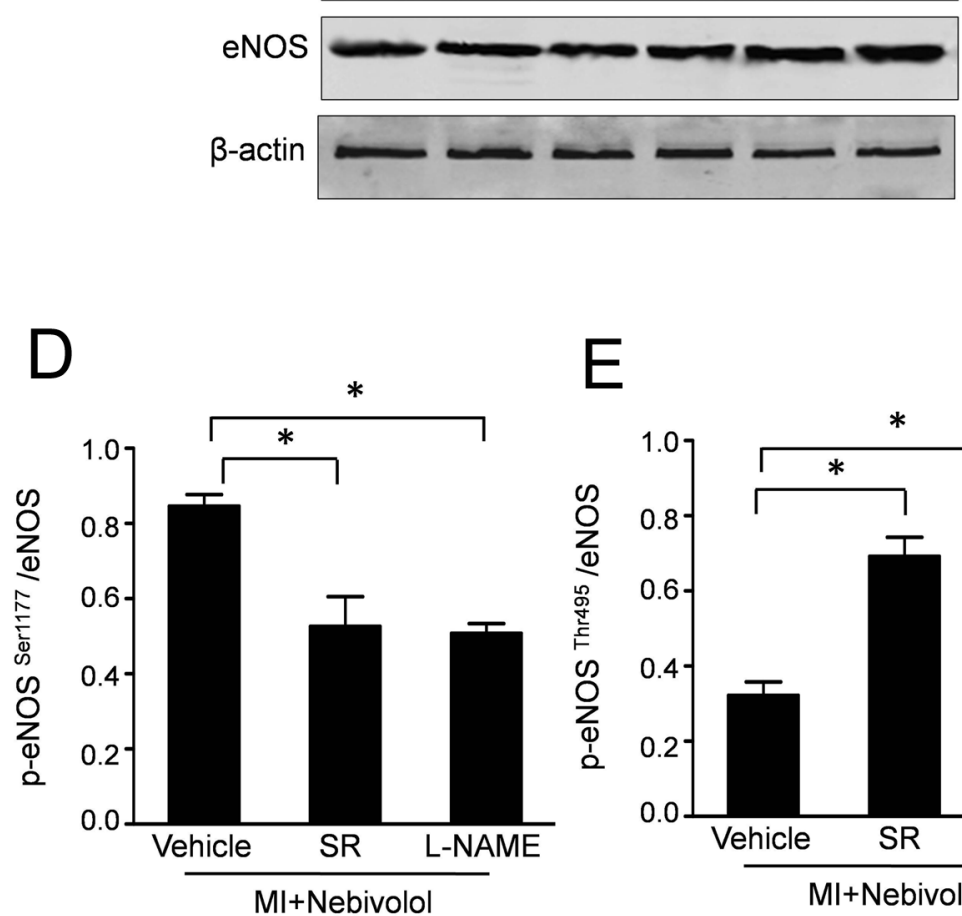
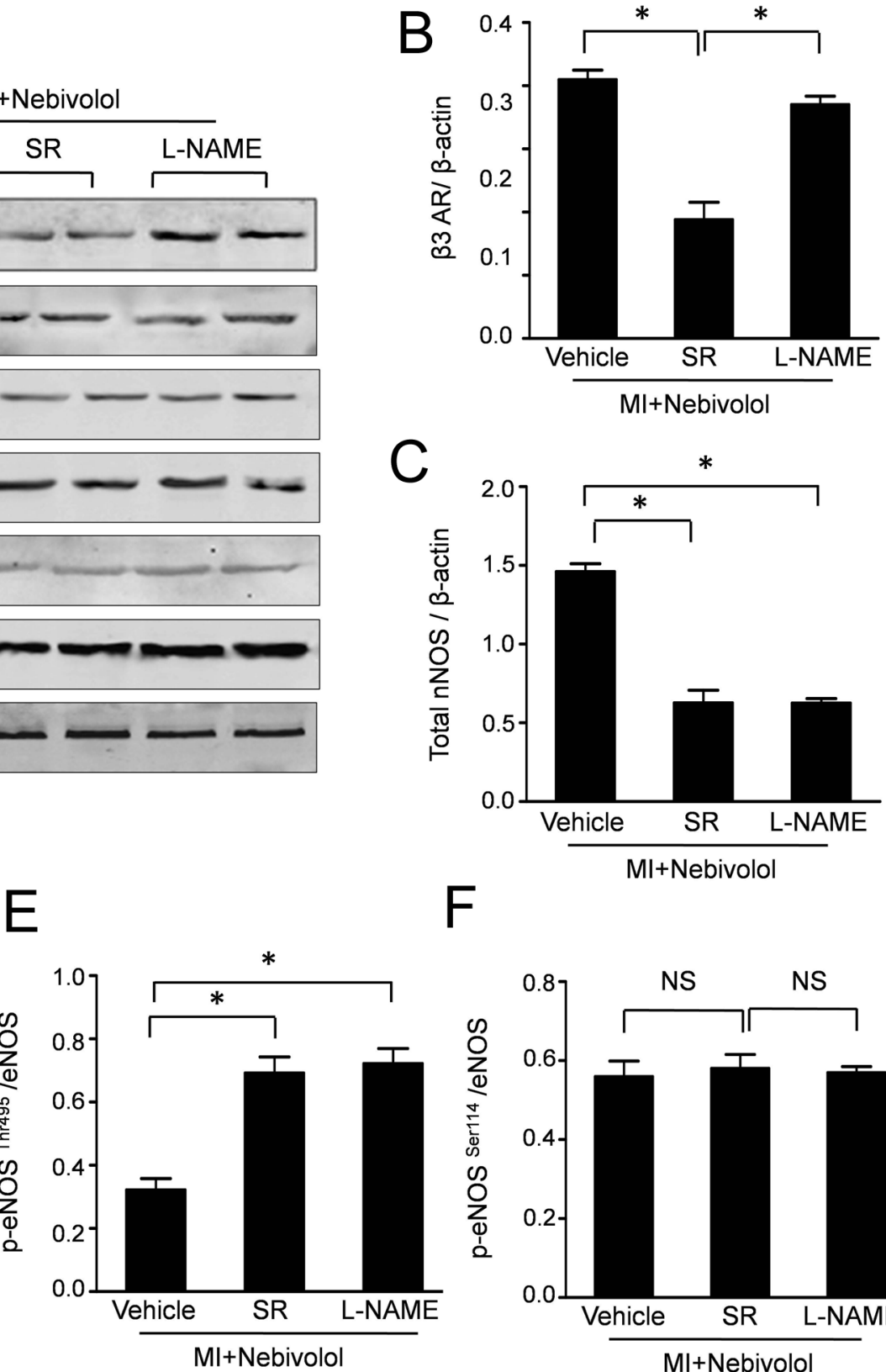

$\mathrm{F}$

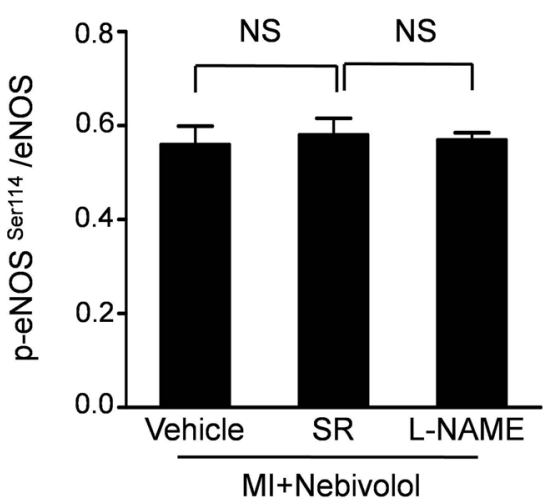

Figure 7. Nebivolol induced $\beta 3$-AR signaling activation was abolished by SR59230A or L-NAME. (a) Representative immunoblots of $\beta 3$ AR, nNOS, $p$-eNOS (Ser1177/Thr495/Ser114) and total eNOS in Nebivolol group, Nebivolol+SR group and Nebivolol+L-NAME group. Semiquantitative

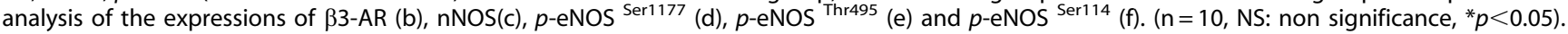
doi:10.1371/journal.pone.0098179.g007

\section{Supporting Information}

Methods S1 Measurement of blood pressure. (DOG)

Table S1 Hemodynamic parameters (heart rate and blood pressure) in different experimental groups. Bpm: beats per minute. Data are mean $\pm S D(n=15) .{ }^{*} \mathrm{P}<0.05$ vs. control group; $\uparrow \mathrm{P}<0.05$ vs. baseline group.

(DOC)

\section{Acknowledgments}

The authors are grateful to Dr. Qiang Li for the Western blot assays and to $\mathrm{Dr}$. Ru Ji for his technological assistance.

\section{Author Contributions}

Conceived and designed the experiments: TH. Performed the experiments: ZZ. Analyzed the data: LD GG. Contributed reagents/materials/analysis tools: ZJ HL LH. Wrote the paper: ZZ. Cell culture: Lina Zhang Lijuan Zhang. Animal model: XL BL XY. 


\section{References}

1. Roger VL, Jacobsen SJ, Weston SA, Goraya TY, Killian J, et al. (2002) Trends in the incidence and survival of patients with hospitalized myocardial infarction, Olmsted County, Minnesota, 1979 to 1994 . Ann Intern Med 136: 341-348.

2. Braunwald E, Bristow MR (2000) Congestive heart failure: fifty years of progress. Circulation 102: IV14-23.

3. Reiken S, Wehrens XH, Vest JA, Barbone A, Klotz S, et al. (2003) Beta-blockers restore calcium release channel function and improve cardiac muscle performance in human heart failure. Circulation 107: 2459-2466.

4. Brixius K, Bloch W, Ziskoven C, Bolck B, Napp A, et al. (2006) Beta3adrenergic eNOS stimulation in left ventricular murine myocardium. Can J Physiol Pharmacol 84: 1051-1060.

5. Tavernier G, Toumaniantz G, Erfanian M, Heymann MF, Laurent K, et al. (2003) beta3-Adrenergic stimulation produces a decrease of cardiac contractility ex vivo in mice overexpressing the human beta3-adrenergic receptor. Cardiovasc Res 59: 288-296.

6. Skeberdis VA, Gendviliene V, Zablockaite D, Treinys R, Macianskiene R, et al. (2008) beta3-adrenergic receptor activation increases human atrial tissue contractility and stimulates the L-type Ca2+ current. J Clin Invest 118: 3219 3227.

7. Rozec B, Gauthier G (2006) beta3-adrenoceptors in the cardiovascular system: putative roles in human pathologies. Pharmacol Ther 111: 652-673.

8. Niu X, Watts VL, Cingolani OH, Sivakumaran V, Leyton-Mange JS, et al. (2012) Cardioprotective effect of beta-3 adrenergic receptor agonism: role of neuronal nitric oxide synthase. J Am Coll Cardiol 59: 1979-1987.

9. Varghese P, Harrison RW, Lofthouse RA, Georgakopoulos D, Berkowitz DE, et al. (2000) beta(3)-adrenoceptor deficiency blocks nitric oxide-dependent inhibition of myocardial contractility. J Clin Invest 106: 697-703.

10. Moniotte S, Kobzik L, Feron O, Trochu JN, Gauthier G, et al. (2001) Upregulation of beta(3)-adrenoceptors and altered contractile response to inotropic amines in human failing myocardium. Circulation 103: 1649-1655.

11. Mercanoglu GO, Pamukcu B, Safran N, Mercanoglu F, Fici F, et al. (2010) Nebivolol prevents remodeling in a rat myocardial infarction model: an echocardiographic study. Anadolu Kardiyol Derg 10: 18-27.

12. Dobre D, van Veldhuisen DJ, Mordenti G, Vintila M, Haaijer-Ruskamp FM, et al. (2007) Tolerability and dose-related effects of nebivolol in elderly patients with heart failure: data from the Study of the Effects of Nebivolol Intervention on Outcomes and Rehospitalisation in Seniors with Heart Failure (SENIORS) trial. Am Heart J 154: 109-115.
13. Karter Y (2007) Nebivolol: more than a highly selective Beta blocker. Recent Pat Cardiovasc Drug Discov 2: 152-155.

14. Maffei A, Di Pardo A, Carangi R, Carullo P, Poulet R, et al. (2007) Nebivolol induces nitric oxide release in the heart through inducible nitric oxide synthase activation. Hypertension 50: 652-656.

15. Zhang Z, Li S, Cui M, Gao X, Sun D, et al. (2013) Rosuvastatin enhances the therapeutic efficacy of adipose-derived mesenchymal stem cells for myocardial infarction via PI3K/Akt and MEK/ERK pathways. Basic Res Cardiol 108: 333.

16. Sun D, Huang J, Zhang Z, Gao H, Li J, et al. (2012) Luteolin limits infarct size and improves cardiac function after myocardium ischemia/reperfusion injury in diabetic rats. PLoS One 7: e33491.

17. Zhang Z, Li W, Sun D, Zhao L, Zhang R, et al. (2011) Toll-like receptor 4 signaling in dysfunction of cardiac microvascular endothelial cells under hypoxia/reoxygenation. Inflamm Res 60: 37-45.

18. Sorrentino SA, Doerries C, Manes C, Speer T, Dessy C, et al. (2011) Nebivolo exerts beneficial effects on endothelial function, early endothelial progenitor cells, myocardial neovascularization, and left ventricular dysfunction early after myocardial infarction beyond conventional betal-blockade. J Am Coll Cardiol 57: 601-611.

19. Aragon JP, Condit ME, Bhushan S, Predmore BL, Patel SS, et al. (2011) Beta3adrenoreceptor stimulation ameliorates myocardial ischemia-reperfusion injury via endothelial nitric oxide synthase and neuronal nitric oxide synthase activation. J Am Coll Cardiol 58: 2683-2691.

20. Rozec B, Noireaud J, Trochu JN, Gauthier C (2003) Place of beta 3adrenoceptors among other beta-adrenoceptor subtypes in the regulation of the cardiovascular system. Arch Mal Coeur Vaiss 96: 905-913.

21. Gauthier C, Tavernier G, Charpentier F, Langin D, Le Marec H (1996) Functional beta3-adrenoceptor in the human heart. J Clin Invest 98: 556-562.

22. Gauthier C, Leblais V, Kobzik L, Trochu JN, Khandoudi N, et al. (1998) The negative inotropic effect of beta3-adrenoceptor stimulation is mediated by activation of a nitric oxide synthase pathway in human ventricle. J Clin Invest 102: 1377-1384

23. Iwakiri Y, Tsai MH, McCabe TJ, Gratton JP, Fulton D, et al. (2002) Phosphorylation of eNOS initiates excessive NO production in early phases of portal hypertension. Am J Physiol Heart Circ Physiol 282: H2084-2090.

24. Martin SR, Emanuel K, Sears CE, Zhang YH, Casadei B (2006) Are myocardial eNOS and nNOS involved in the beta-adrenergic and muscarinic regulation of inotropy? A systematic investigation. Cardiovasc Res 70: 97-106. 